$\$$ sciendo

\title{
Do Honeybees Have Concepts?
}

\author{
Bernardo Aguilera Dreyse \\ University of Sheffield
}

Disputatio Vol. 4, No. 30

May 2011

DOI: 10.2478/disp-2011-0002

ISSN: 0873-626X 


\title{
Do honeybees have concepts?
}

\author{
Bernardo Aguilera Dreyse \\ University of Sheffield
}

\begin{abstract}
Can animals think? In this paper I address the proposal that many animals, including insects such as honeybees, have genuine thoughts. I consider one prominent version of this view (Carruthers 2004; 2006) that claims that honeybees can represent and process information about their environments in a way that satisfies the main hallmarks of human conceptual thought. I shall argue, however, that this view fails to provide convincing grounds for accepting that animals possess concepts. More precisely, I suggest that two important aspects of conceptual thought, viz., concept individuation and the generality constraint, are not satisfied.
\end{abstract}

\section{Keywords}

Animal cognition, concepts, modularity, concept individuation, generality constraint.

\section{Introduction}

The long standing debate about whether animals can think has been fuelled in recent years by scientific research that provides strong evidence that the cognitive processes of many animal species are computational. Behaviours that at first sight could appear as immediate responses to environmental contingencies, after careful observation and experimental procedures have shown to be governed by structured representations following complex computational algorithms. Examples come from diverse parts of the animal kingdom, including insects, birds and mammals.

These findings have been received with enthusiasm by advocates of a particular view of animal cognition (Carruthers 2006), which embraces a version of the computational theory of mind (CTM) and the massive modularity hypothesis (MMH). In short, this view holds that animals process information about their environment by means of computation, and that their cognitive architecture is mainly modularised into domain specific processors. In this paper I shall discuss the 
claim, put forward by proponents of this view, that most animals have concepts ${ }^{1}$, and that those concepts can combine forming (propositional) thoughts. In particular, I deal with the case of honeybees, for two reasons. First, because their behaviour has been extensively studied and there is general consent that they carry out computations, and secondly because it is particularly provocative to suggest that they can think. If it turns out that honeybees have concepts and thoughts, it appears convincing that this capacity is widespread in the animal kingdom.

In this paper I shall assume that CTM gives a plausible account of human concepts and thoughts and, likewise, that there are no reasons in principle to reject the idea that cognitive architecture is massively modular. However, I will argue that when these ideas are deployed for the case of honeybee cognition, in the way put forward by Carruthers, they fail to provide convincing grounds for the possession of concepts. More precisely, I shall claim that two main features of conceptual thought, i.e. concept individuation and the generality constraint, are not always satisfied.

This paper is structured in the following way. In section 2 I sketch the basic tenets of CTM and MMH, and in section 3 I explain how CTM gives a plausible account of conceptual thought, addressing some common objections. These sections are intended to give a general background about the views that have inspired the proposal about animal cognition that is criticised further on in the paper. Section 4 gives a brief exposition of current research in honeybee behaviour in order to make clear how it strongly suggests that they are computational systems. In section 5 I explain the claims put forward by authors who interpret this symbolic processing as a form of conceptual thought, and then in sections 6 and 7 I present my arguments against that view. Lastly, section 8 gives some final remarks.

\footnotetext{
${ }^{1}$ A terminological note: In philosophical usage, 'concepts' are generally understood as abstract entities, however in psychology the term is used to designate mental representations (Margolis \& Laurence 2007). In this paper I will follow the psychological usage, but understanding particular mental representations as concept tokens, that instantiate mental representation types. Whenever I refer to concept tokens I shall use italics, and when referring to concept types I shall write them in capitals.
} 


\section{Concepts, Computation and Modularity}

The mind provides us with a meaningful perspective of the world, and much of that job is carried out through our capacity to conceptualise what our perceptual systems bring to our minds (cf. Crane 2001). Concepts are the main building blocks of our world view and therefore they are generally considered as one of the main features of the mind. Concepts also make up thoughts, which allow us to have mental states that interact causally to produce intelligent behaviour. I leave open the possibility that a mind could be defined by states that are non-conceptual, such as perceptions or emotions. However, for the purposes of this paper I shall be interested in the common view that minded creatures can think, and that thoughts are constituted by concepts.

This view is compatible with CTM, which has been the dominant approach of how the mind works over the past four decades. The CTM has two basic tenets. One is that the mind is a representational system. That means that the mind picks up information about the environment and encodes it as mental representations. This information is made available by perceptual systems, and can be stored in memory for future processing (Sterelny 1990). The second tenet is that mental representations are processed following computational (i.e. algorithmic) steps. This means that these processes are performed in ways only responsive to the formal properties of the representational states, whilst their contents (i.e. what they mean) are preserved along the computational steps without playing any causal role in the process (Haugeland 1981). One of the most influential articulations of CTM has been by Fodor $(1975 ; 1987)$. Since he is often quoted by the proponents of animal cognition, I will focus on his account of CTM for the rest of this paper.

One of the main contributions by Fodor to CTM was to make explicit the idea that the mind must have an inner medium of representation that carries out the computations. He also claimed that the properties of productivity and systematicity of thought could only be explained if this inner medium has the compositional structure of a language. For that reason, he proposed that the mind has a language of thought (LOT). According to this view, thinking consists in entertaining sentences in LOT. Words in LOT express concepts, and sentences express propositions. LOT is where thought and its properties (i.e. syntactic and semantic) are situated. Its basic structure is 
supposed to be innate, and thus not dependent on learning a language. As noted above, this idea has important implications for animal cognition, since it states that thinking is not a capacity derived from the possession of a natural language, leaving open the possibility that non-linguistic creatures could think.

However, it is important to be careful when attributing concepts to animals. For example, pigeons can be trained to sort pictures into categories of tree or person, but these findings do not warrant the conclusion that they have concepts. Pigeons may be just grouping together common visual elements into a single internal representation, without being able to make further recognitional distinctions and inferences that are characteristic of possessing abstract concepts such as those of a tree or a person (Allen \& Hauser 1996). I shall say more about these capacities and the individuation of concepts in the next section. For the moment, it suffices to say that the view criticised in this paper claims that some of the internal representations of honeybees are not just trivial forms of information processing, but also meet some of the relevant criteria for concept attribution.

The MMH is a claim about cognitive architecture. The main idea is that the mind does not work as a single, domain-general system, but has several functionally distinguishable modules that process domainspecific information and work quickly and rather isolated from one another. Initial accounts of cognitive modules restricted their processing to perceptual and motor information. However, proponents of MMH have proposed that mental processes involving thoughts and reasoning are also modular (Cosmides \& Tooby 1994, Pinker 1997).

According to $\mathrm{MMH}$, the modular parcellation of cognitive capacities constitutes an extremely common evolutionary feature that enhanced the adaptability of organisms by permitting them to deal more efficiently with their environments (Carruthers 2006). That explains why the animal mind is supposed to be massively modular. Some empirical evidence has been put forward to defend this claim. To give one example, the navigational capacities of many animals, including rats and birds, have been shown to be modular (see Shettleworth 1998 for a review). They have been studied in artificial environments that offer limited kinds of information that can be used by them to orientate. Animals proved to be able, not only to use these different environmental clues to navigate, but to deploy them in a way that requires computation, such as vector addition or template matching. However, some kinds of information appear to be per- 
ceived and used independently, without the capacity to integrate it with other visual clues. All this suggests that they process the various kinds of spatial information by dedicated cognitive modules, that exhibit the hallmarks of domain-specificity, computational processing and isolation.

\section{Conceptual thought in CTM: content and individuation of concepts}

According to CTM, thoughts are sentences in LOT and concepts are the elements from which they are constructed. When an agent is thinking, chains of propositions are tokened in her mind, one leading to the other following algorithmic steps that are sensitive to the syntactic properties of LOT. So, for example, an agent could think:

When it's raining, there are no rabbits in the meadow

Now it's raining

So, there are no rabbits in the meadow

Here, the propositions have a syntactic structure that can be recognised by the system (i.e. the brain) as an instance of modus ponens, and then processed in a way that mirrors the logical structure of the argument. The thought can be carried out mechanically, independently of the content of the concepts involved in it. This suggests that thought can be viewed as a purely syntactic procedure.

This idea of mechanised thinking is at the core of CTM. It has many advantages, one of them being how the logical structure of reasoning could be implemented in a digital computer. However, it has the counterintuitive consequence that what-the-thought-is-about does not appear to play any causal role in the thinking process. In the previous example, we could replace rabbits for foxes, and the thinking process will still be the same (i.e. an instance of modus ponens specifiable purely by syntax), however, of course it is not the same to think about rabbits as it is to think about foxes. Moreover, how could a creature build up a perspective on the world with a representational system that is purely syntactic? How could concepts be regarded as meaningful then?

The response of CTM to these questions is that they simply never said that semantics could be ignored, or that it could be reduced to syntax (Horst 2009). Most proponents of CTM have divorced them- 
selves from an extreme view about the 'autonomy' of a syntactic language, perhaps advocated by early developers of artificial intelligence, a view that would allow concepts to become meaningful just in virtue of local intra-linguistic manipulations. Instead, they claim that the mind is a large syntactic system ${ }^{2}$ capable of interacting causally with the environment, in a way that can be described as denoting objects and properties in the world (Rey 1997, Haugeland 2003). On his view about animal cognition, Carruthers $(2004$; 2006) seems to agree with these constraints on being a genuine thinker, adding the idea that this syntactic system should mirror a belief/desire cognitive architecture.

Returning to the issue about the meaning of a concept, CTM claims that it is determined by its content, which is fixed from 'outside' the domain of thought by the input and output causal relations that concept has with the external world. For example, what makes an agent to instantiate the concept of RABBIT is that she has been caused to think about rabbits every time there has been a causal connection between rabbits and her perceptual systems. In other words, the interaction of the agent with the word gives the concepts meaning, which is then preserved along the computational processes where the concept takes part. This account of conceptual content is usually called 'causal theory of content'. There is controversy about how to precisely determine content, and several theories are available. However, for the purposes of this paper, suffice it to say that causal factors in determining content are dominant among theorists of CTM (Rey 1997).

It is important to note that even though conceptual content is independent from the syntactic structure of thought, this is not the case with concept individuation. Two concepts may share their contents (i.e. have the same extension), but differ in two further aspects. One of them is the expression in LOT where they are instantiated, what is usually called the 'mode of presentation'. For example, an agent could think about water both tokening the expression water and $\mathrm{H}_{2} \mathrm{O}^{3}$, which constitute two modes of presentation for the same content. A

\footnotetext{
${ }^{2}$ In fact, this can be considered a version of what Searle 1980 calls 'the systems reply' to his famous Chinese Room argument.

${ }^{3}$ To simplify the exposition, I am using English words as expressions of LOT. But LOT, at least according to CTM, does not correspond to any natural language.
} 
second aspect is the inferential role of these expressions. Two concepts can share the same content, though differing in their causal effects on other thoughts and behaviour. So when an agent thinks tokening the LOT expression water, she may be lead to think about drinking, but when tokening $\mathrm{H}_{2} \mathrm{O}$ may be caused to think about chemistry. Both concepts have the same meaning, but differ in their modes of presentation and causal roles.

To sum up: when concepts are instantiated in mental states (i.e. as concept-tokens), they are individuated by their content, mode of presentation and inferential role. There is controversy about how to specify causal roles (cf. Fodor 1992: ch.6 and Margolis \& Laurence 2007), but that should not bother us here. The main point I want to present is that when an agent instantiates a concept (-type) in her mind, she has a mental representation with a content that express the same meaning, tokened in a particular expression in LOT, and with a particular inferential role over the rest of LOT. Any account about the cognitive significance that is implied with the possession of a concept should consider these three aspects of concept individuation.

Though the picture of the mind given by CTM has some controversial issues, it still appears suitable for the purposes of cognitive psychology at least for three reasons. First, through the idea of an internal language and mechanisms to fix content, it provides a plausible account of how the mind could make up a meaningful perspective on the world. Second, it explains how this perspective could be realistically constructed, by showing how mental states could be instantiated in an internal medium of representation. This allows us to treat the agent as a genuine thinker with real and casually efficacious mental states, and also helps to solve the 'mode of presentation problem' (i.e. explaining how an agent could have two different thoughts about the same thing, by using different LOT expressions).

Finally, a third advantage of this view is that it can serve the purposes of a scientific psychology, by giving an account of how the contents of structured mental states can take part in the mental life of an organism. LOT provides the cognitive vehicles for causally efficacious sequences of thoughts, whilst their inferential roles can describe computational patterns that instantiate principles of rationality. In other words, it allows a scientific explanation of how cognitive agents behave in virtue of their intentional mental states (i.e. beliefs, desires, etc.). This is particularly important for this essay, since philosophers of cognitive ethology have embraced a similar view for animal psy- 
chology (see Allen \& Bekoff 2006 and Carruthers 2004; 2006). They claim that a mentalistic framework like that used by cognitive psychology (i.e. folk-psychology) can be applied to explain animal behaviour, attributing many animals with structured thoughts that interact causally according to rational patterns.

\section{Honeybees as computational systems}

In this section I will give a brief review of some complex behaviours that have been studied in honeybees in order to show how plausible it seems to claim that they are endowed with computational states and processes.

As is well known, honeybees have notable navigational capacities that make them able to fly from their hives to sources of food and then return. Sometimes they rely on landmarks to orientate, whilst they also use dead reckoning (calculate their position by estimating the direction and distance travelled). They exploit the solar azimuth as a directional referent, being able to estimate its position in the sky at different times of the day in order to set and hold a compass course (Collett \& Collett 2002). More surprisingly, they can integrate this information and use it flexibly. For example, in some experiments bees were captured after feeding and carried in a dark box to an unfamiliar releasing point. When released, they initially continued to fly the course they were on when captured, but they soon recognised they were lost, and began an extensive search until they found a familiar landmark. Then, they were able to fly straight to their hives in a vector they have never flown before (Menzel et al. 2000). These experiments suggest that honeybees can represent many features of their environments and integrate them with stored information about distance and direction relative to their hives.

Another remarkable fact about honeybees is their communicational capacities. Foraging bees transmit information about food resources to other bees via different kinds of dances they perform inside the hive (Gould \& Gould 1996). Some features of the dance such as the angle of movement as measured from the vertical, and the number of 'waggles' they made at some point of the dance, convey information about the expected direction of the sun for the time of the day, and the distance of the food source. The bees in the hive are not just able to integrate the communicated information and fly to the 
food, but can also evaluate it along a number of dimensions. For example, they are less likely to fly to distant sources of food, and show preference for rich sources of food.

These findings suggest that the behaviour of honeybees cannot rely exclusively on fixed action patterns, or be conditioned responses to stimuli. Instead, they seem able to form complex and structured representations of their environments, including information about distance, time, direction and location. They can also transmit this information and use it in a rather flexible and systematic way. Plausibly, many authors have claimed that the best explanation for these complex behaviours is that honeybees can carry out computational processes over causally efficacious and structured representations (Carruthers 2006, Gallistel 2009, Tetzlaff \& Rey 2009).

\section{Honeybees as thinking creatures}

Through several writings, Carruthers (2004; 2006; 2009) has given a detailed defence of the computational capacities and the massive modularity of animal cognition. Among them are writings on honeybees, whose striking behavioural complexities I summarised above. He also moves a step forward in claiming that honeybees have conceptual thought, according with the framework of CTM. I shall summarise his view and then present my arguments against it in the following sections.

Carruthers argues that the capacity of certain animals to represent specific features of their environment and to process them following algorithmic steps, constitutes a genuine form of means/ends reasoning. He contrasts it with forms of associative conditioning or innate releasing mechanisms, which cannot explain the flexibility and complexity of certain behaviours (e.g. those of the honeybees presented above). On the contrary, many animal behaviours are mediated by cognitive processes that involve explicit representations and purposeful reasoning. He goes on to claim that these processes can be characterised in terms of belief-states and desire-states that are discrete, structured, and causally efficacious in virtue of their structural properties.

A great part of the force of Carruthers' argument rests on two assumptions. The first is that CTM works for human beings, an assumption that I shall take for granted for the purposes of this paper. Sec- 
ond, and more importantly for present purposes is that the difference between human and animal cognition is basically a matter of complexity and not of kind. He claims that there are no reasons a priori to impose human standards to define 'mindness', and that what matters for having a mind is to possess the right cognitive architecture: a compositional medium of representation able to structure internal states that can interact causally through basic practical inferences to select and guide behaviour. But, why regard these internal states as genuine beliefs and desires?

The response given by Carruthers 2004 is that, after studying animal behaviour, we can infer that those internal states have a structure that resembles that of beliefs and desires, both in their propositional form and in the way they interact following the characteristic roles of beliefs and desires in practical reasoning. So, by virtue of their resemblance to our own internal states and folk-psychology, he argues that these internal states can be externally (and realistically) characterised as beliefs and desires, and therefore structured by concepts.

As previously noted, Carruthers' idea of animal cognition also involves claims about massive modular architecture. The computational systems of animals are supposed to be organised in cognitive modules, and that implies that the representations and computations the animal carries out are distributed into separated, domain-specific and rather isolated units. For example, honeybees seem to use distinct modular systems to navigate inside or outside their hives (Carruthers 2009). When inside the hive they orientate themselves using gravity-based and olfactory cues, whilst they rely on solar bearings when outside it. It is not that they choose between one navigational system or another. According to MMH, honeybees do not represent those systems as alternative sets of spatial representations ${ }^{4}$, but they activate one or another when the relevant input is present. So, if honeybees are thinkers, they deploy different ways of thinking about their environment depending on which module they are using. Those differences are principally related with the representational vehicles they deploy (e.g. from distinct perceptual formats), and the inferential roles they occupy (i.e. directed to different domain-specific tasks).

\footnotetext{
${ }^{4}$ Because they lack second order beliefs. In Carruthers (2002), the author defends his view that cross-modular cognitive integration of thoughts is restricted to linguistic creatures.
} 


\section{First argument against Carruthers' proposal: concept individuation}

Now I shall argue that the view that honeybees have conceptual thought, as explained in the preceding section, has several problems. In particular, I argue that it becomes implausible to state that honeybees can individuate and use the concept HIVE. Since this concept denotes an essential feature of their environment, it is hard to see how they could be treated as genuine thinkers if they lack it.

My arguments outline some problems associated with the idea that animals with a simple cognitive system and a massively modular architecture could individuate concepts. Recall the two navigation modules that honeybees have for orientating inside or outside their hives. They should be able to entertain the concept of HIVE in both cases, presumably in different representational formats, one based on gravitational and olfactory cues, whilst the other based on visual cues. Also, they should be able to combine this concept with others, in order to form propositional thoughts. According with the framework of MMH, instances of the concept of HIVE should coexist within each module with other concepts that concern the specific computations that the module was designed to perform. For example, suppose that the navigational module for outside the hive has the concept of BLUE, whilst the module for inside does not. Instead the module for navigating inside the hive has the concept of WAX-ODOUR, absent in the other module. This observation leads to the conclusion that the honeybee would only be able to think the hive has wax-odour when it is inside the hive, whilst the thought the hive is blue could only be entertained when outside it.

Does that mean that the honeybee has two different concepts of HIVE, one for each module? If we recall how CTM defined the semantic properties of LOT, we could give a tentative response: they are instances of the same concept, since they both have the same extension, i.e. they are both about hives. However, their mode of presentation and inferential role in the propositions they constitute must be different, since each module has a domain-specific set of representations and causal roles. They respond to specific input and output channels and carry out the computations specified by the function of the module. So the honeybee appear to be instantiating 
two different concepts, and this could be seen as a problem when individuating them as tokens of the same concept of HIVE.

However, Carruthers sees this situation as unproblematic, suggesting that animals do not have a single LOT, but several LOTs, one for each module. That means that the honeybee may turn out to have many modules which can think about hives, but do it in radically different ways, as if there were different languages that cannot understand each other. However, I believe that this idea does not work. Let me restate some ideas about concept individuation to then expose my arguments.

What fixes the content of HIVE is its extension. Two agents share the content of HIVE if hives in the world causally co-vary with instances of HIVE in their heads. But, as I explained in section 3, concept-tokens are not individuated just by their contents. Content attribution depends on their extension, whilst concept attribution also depends on their LOT expression and inferential role. So, to some extent the meaning of a concept is independent of its inferential role, but that does not imply that inferential roles are irrelevant to determine whether a system is really instantiating a concept. According to CTM, LOT tokens realise concepts thanks to their place in a causal network that connects them to the world in the appropriate way, in the sense that the semantic properties of the tokens are preserved along the computational processes of the system. This imposes a constraint on the internal coherence on the system, which makes possible the fact that not any interpretation of the semantic properties of concepts could 'make sense' (Haugeland 1981). Otherwise, if any interpretational scheme could make sense of what the system's tokens are about, concept attribution would become something trivial.

This idea of internal coherence is what makes the individuation of concepts problematic in the present case. Even if an agent could think about the same thing in different ways, and thus individuate the same concept-type in several concept-tokens, there should be a consistent relation between their inferential roles, at least making it plausible to justify concept possession instead of simple forms of categorisation (cf. section 2). If an agent happens to think about hives in radically different ways, we may be justified to doubt whether this agent could possess the concept of HIVE in any relevant sense. And this seems to be the case of honeybees, since each module processes information about hives through representational vehicles and computations that were designed for specific tasks, that may be different and even 
contravene one another. If nothing ensures some degree of internal coherency within the conceptual system of an agent, to treat modular processes as instantiating genuine concepts strikes me at least as problematic.

It is important to remark that I do not intend to raise general scepticism about how concepts could be instantiated in a massively modular mind, or be shared among different people. For instance, it is plausible to suggest that in the case of human beings the faculty of language provides a medium for conceptual identity (or similarity) within the mind and also among people who share a natural language. But this is not, of course, the case of honeybees, and so it is unlikely that their cognitive architecture could have the resources to deal with the concerns about concept individuation presented in this paper.

\section{7- Second argument against Carruthers' proposal: the Generality Constraint}

My second argument is also related to some consequences of MMH in the individuation of concepts, but this time I focus on the generality constraint (GC). The GC is often assumed as an essential characteristic of conceptual thought, and was first stated by Evans as follows:

We cannot avoid thinking of a thought about an individual object $\mathrm{x}$, to the effect that it is F, as the exercise of two separable capacities; one being the capacity to think of $\mathrm{x}$, which could be equally exercised in thoughts about $\mathrm{x}$ to the effect that it is $\mathrm{G}$ or $\mathrm{H}$; and the other being a conception of what it is to be F, which could be equally exercised in thoughts about other individuals, to the effect that they are F. (Evans 1982: 75)

The main idea is that genuine thinkers should be capable of producing and entertaining an unbounded set of novel well-formed combinations of concepts. This capacity is closely related with what has been called the systematicity and productivity of thought, which have been proclaimed by CTM theorists as elemental features of thought. In Fodor's words:

Productivity and systematicity are also universal features of human thought (and, for all I know, of the thoughts of many infra-human crea- 
tures). There is no upper bound to the number of thoughts that a person can think. (Fodor 1994: 106-7)

Moreover, CTM offers one of the most compelling explanations about the cognitive mechanisms that underlie these features, based on the compositional nature of LOT (see section 2). So the GC can be safely regarded as a hallmark of thought that honeybees should fulfil if they have genuine concepts.

Now suppose that the perceptual apparatus of the honeybee is able to discriminate between three colours: green, yellow and red, and that this capacity is deployed in a module for flower recognition which does not have hive among its repertoire of concepts (this example is fictional, but serves to exemplify some possible consequences of the $\mathrm{MMH}$ ). Also, suppose that the navigational module for outside the hive mentioned earlier includes among its domain-specific repertoire of representations for colour just green and yellow, but not red. So, among the operations of this module the honeybee might be able to combine the concept of HIVE with the concepts of GREEN and YELLOW, forming the thoughts the hive is green and the hive is yellow. However, she will not be able to think the hive is red. This appears to violate the $\mathrm{GC}$.

Carruthers $(2004 ; 2009)$ has defended the conceptual capacities of animals, based on their apparent capacity to form thoughts with compositional structure. He acknowledges that given the restrictions derived from a modular architecture, honeybees may be unable to meet the GC. A clear case is that they have limited productivity, since their cognitive architecture prevents their concepts to combine with others outside their own modules. Carruthers plausibly makes the point that the capacity to creatively form an unbounded set of new combinations between concepts appear to be a particular human capability, that does not seem to be necessary for concept possession. But he does claim that genuine thought must be at least partially systematic. He states his position with what he calls a 'weak' version of the GC, defined as follows:

If a creature possesses the concepts $F$ and $a$ (and is capable of thinking $\mathrm{Fa}$ ), then for some other concepts $G$ and $b$ that the creature could possess, it is metaphysically possible for the creature to think Ga, and in the same sense possible for it to think Fb. (Carruthers 2009: 97) 
Following this definition, for a creature to satisfy the weak GC we should expect it to be able to make at least some combinations between the concepts it possesses, as opposed to a 'strong' version of the GC where it should be capable to think all possible combinations. So in the previous example, even though honeybees cannot deploy their concept of HIVE to think the hive is red, the fact that they can think the hive is green and the hive is yellow shows that they have the capacity to recombine their concepts, at least in a modest way that satisfies the weak GC.

But, why should we accept this weak version of the GC? Is it too modest? Carruthers argues that it satisfies what he takes to be the core of concept possessing: compositionality. This is the capacity to have thoughts that are structured in a way in which its components can be detached from their current form to re-structure at least some other thoughts. To have the capacity to make all possible combinations of thoughts constitutes an ideal, he suggests, that perhaps only humans can get close to achieving.

I find his defence of the weak GC unconvincing. Even though it works as a constraint on compositional structure, it is too weak as a constraint on genuine thinking as is the purpose of the GC. A creature who is able to entertain concepts should be able to detach them from their current inferential roles, in order to then deploy them in new compositional thoughts. But let us imagine a module with a fixed architecture consisting on a few combinable concepts. It would satisfy the weak GC, however nothing implies that its concepts could be detached from their current roles. If the algorithms carried out by its cognitive machinery are innately specified and thus hardwired within the margins of the module, the inferential roles of its concepts are not modifiable, even if the concepts appear mirroring certain combinations.

However, perhaps a more serious problem with Carruthers' proposal comes again as a consequence of the massive modular architecture of honeybee cognition. Following its original formulation, the $\mathrm{GC}$ is intended to ensure that when a creature really has the concept $F$, we are committed to the view that when it has any thought that deploys this concept (e.g. Fa, Fb, etc.) it is exercising the same conceptual capacity (see Evans 1982: 101-105). However, this is does not seem work with honeybees. Let me explain this with an example.

Recall the previous example of the two modules for flower recognition and for navigation. The honeybee would be able to think the 
flower is yellow in the first module, whilst the hive is yellow in the second. Contrary to what the GC proclaims, the conceptual capacities deployed to think about the concept of YELLOW in both cases are different, thus raising doubts about whether the insect is really able to entertain the concept of YELLOW. It could be argued that both modules share the same conceptual capacities, but the nature of cognitive modules seems to count against this idea. Cognitive modules are often conceived as 'mental organs' in analogy with the organs of the body, since they evolved functionally specialised mechanisms in same way as the heart or the lungs (Pinker 1997). It is a natural consequence of this specialisation that the functions performed by these organs correspond to distinct biological capacities, and in the same sense the functions performed by each module can be regarded as the product of distinct cognitive capacities.

\section{Final remarks}

As I have stated since the beginning, my purpose in this paper has not been to criticise the main tenets of CTM or MMH. They could be perfectly true, and some version of them suitable for animal cognition. My point has been to argue that the requirements for conceptual thought are not fulfilled in a model that simply conjoins both views without further refinements. It has also been my purpose to direct my arguments to a simple cognitive architecture, as seems to be the case of honeybee cognition. More sophisticated versions of MMH, which may incorporate massive conceptual networks and/or mechanisms for cognitive integration, may well be immune to the arguments raised in the present paper.

It is always tempting to attribute a belief-desire psychology to animals, and, without doubt, the evidence of their computational capacities make them good candidates to be thinkers. However, whether this evidence alone is enough to account for the conceptual nature of their representations is far from clear. It has been the purpose of this paper to show that Carruthers' account of honeybee concepts presents at least two problems. A conclusion could be, to put it roughly, that there is good evidence to regard honeybees as sophisticated computers, however not good reasons to regard them as having thoughts and concepts. 
Perhaps one of the main limitations of $\mathrm{MMH}$ to give a plausible picture of a mind is that it goes against the intuitive view of the mind as a unitary perspective on the world. As noted in section 3, in order to sound plausible CTM needs to claim that the meaning of concepts does not reside in local computational pathways, but in processes that are part of a whole computational system connected with the external world. It is hard to see how concepts that are enclosed in modules and therefore cannot interact with those from other modules could be regarded as part of such a unitary conceptual system.

A plausible alternative, I believe, could still hold that animal cognition is massively modular, but claim that genuine minds emerged when second-order representations (or metarepresentations) evolved in animals. This could have provided a cross-modular medium to detach the split repertoire of representations contained in modules, and integrate them into a unitary representational system, that gets closer to an intuitive picture of what a mind is. Some authors have suggested that metarepresentational capacities are present in just a few highly intelligent animals, such as some primates (Sperber 2000). However, others have proposed that metarepresentations could be wide spread in the animal kingdom, probably under a nonpropositional representational format (Bermúdez 2009, Proust 2009). The issue about whether metarepresentations are a necessary condition for having a mind (and therefore genuine concepts), or when they appeared in phylogeny, goes beyond the purposes of this paper, however.

Bernardo Aguilera Dreyse bedobardo@gmail.com

\section{References}

Allen, Colin \& Bekoff, Marc. 1997. Species of Mind: The Philosophy and Biology of Cognitive Ethology. Cambridge MA: MIT Press.

Allen, Colin \& Hauser, Marc. 1996. Concept Attribution in Nonhuman Animals: Theoretical and Methodological Problems in Ascribing Complex Mental Processes. In Readings in Animal Cognition, edited by M. Bekoff \& D. Jamieson. Cambridge MA: MIT Press, 47-62.

Bermúdez, José Luis. 2009. Mind reading in the animal kingdom. In The Philosophy of Animal Minds, edited by R. Lurz. Cambridge: Cambridge University Press, 145-164. 
Carruthers, Peter. 2002. The cognitive functions of language. The Behavioral and brain sciences, 25(6): 657-74.

Carruthers, Peter. 2004. On Being Simple Minded. American Philosophical Quaterly, 41(3): 205-220.

Carruthers, Peter. 2006. The Architecture of the Mind. New York: Oxford University Press.

Carruthers, Peter. 2009. Invertebrate concepts confront the generality constraint (and win). In The Philosophy of Animal Minds, edited by R. Lurz. Cambridge: Cambridge University Press, 89-107.

Collett, T. S., \& Collett, M. 2002. Memory use in insect visual navigation. Nature reviews. Neuroscience, 3(7): 542-52.

Cosmides, Leda \& Tooby, John. 1994. Origins of domain specificity: the evolution of functional organization. In Mapping the mind: Domain specificity in cognition and culture, edited by L. A. Hirschfeld and S. A. Gelman. Cambridge: Cambridge University Press, 85-116.

Crane, Tim. 2001. Elements of Mind. New York: Oxford University Press.

Evans, Gareth. 1982. The Varieties of Reference. Oxford: Oxford University Press.

Fodor, Jerry. 1975. The Language of Thought. Cambridge MA: Harvard University Press.

Fodor, Jerry. 1987. Psychosemantics: The Problem of Meaning in the Philosophy of Mind. London: Bradford.

Fodor, Jerry. 1992. A Theory of Content and Other Essays. Cambridge MA: MIT Press.

Fodor, Jerry. 1994. Concepts: a potboiler. Cognition, 50(1-3): 95-113.

Gallistel, Charles Ransom. 2009. The foundational abstractions. In Of Minds and Language: A Dialogue with Noam Chomsky in the Basque Country, edited by M. Piattelli-Palmirini, J. Uriagereka, \& P. Salaburu. New York: Oxford University Press, 58-73.

Gould, James \& Gould, Carol Grant. 1998. The Honey Bee. New York: Scientific American Library.

Haugeland, John. 1981. Semantic Engines: An Introduction to Mind Design. In Mind Design: Philosophy, Psychology, and Artificial Intelligence, edited by J. Haugeland. Cambridge MA: MIT Press, 1-34.

Haugeland, John. 2003. Syntax, Semantics, Physics. In Views Into the Chinese Room: New Essays on Searle and Artificial Intelligence, edited by J. M. Preston \& M. A. Bishop. New York: Oxford University Press, 379-392.

Horst, Steven. 2009. The Computational Theory of Mind. In The Stanford Encyclopedia of Philosophy, edited by E. Zalta. Retrieved from http://plato.stanford.edu/archives/win2009/entries/computational-mind. 
Margolis, Eric \& Laurence, Stephen. 2007. The Ontology of Concepts-Abstract Objects or Mental Representations? Noûs, 41(4): 561-593.

Menzel, R., Brandt, R., Gumbert, a, Komischke, B., \& Kunze, J. 2000. Two spatial memories for honeybee navigation. Proceedings. Biological sciences / The Royal Society, 267(1447): 961-8.

Pinker, Steven. 1997. How the Mind Works. New York: Norton.

Proust, Jöelle. 2009. The representational basis of brute metacognition: a proposal. In The Philosophy of Animal Minds, edited by R. Lurz. Cambridge: Cambridge University Press, 165-183.

Rey, Georges. 1997. Contemporary Philosophy of Mind: A Contentiously Classical Approach. Oxford: Blackwell.

Searle, John. 1980. Minds, Brains and Programs. The Behavioral and Brain Sciences, 3: 417-24.

Shettleworth, Sara J. 1998. Cognition, Evolution, and Behavior. New York: Oxford University Press.

Shettleworth, Sara J. 1998. Cognition, Evolution, and Behavior. New York: Oxford University Press.

Sperber, Dan. 2000. Metarepresentations in an Evolutionary Perspective. In Metarepresentations: A Multidisciplinary Perspective, edited by D. Sperber. New York: Oxford University Press, 117-138.

Sterelny, Kim. 1990. The Representational Theory of Mind: An Introduction. Oxford: Basil Blackwell.

Tetzlaff, Michael \& Rey, Georges. 2009. Systematicity and intentional realism in honeybee navigation. In The Philosophy of Animal Minds, edited by R. Lurz. Cambridge: Cambridge University Press, 72-88. 